R. J. Cohen and W. T. Sullivan, III, eds.

\title{
Observing Conditions from 1988 to 1999 at Huairou Solar Observing Station
}

\author{
Yuanyong Deng ${ }^{1,2}$ and Yihua Yan ${ }^{1,2,3}$ \\ ${ }^{1}$ Beijing Astronomical Observatory, CAS, Beijing 100012, China \\ ${ }^{2}$ National Astronomical Observatories, CAS, Beijing 100012, China \\ ${ }^{3}$ CAS-Peking University joint Beijing Astrophysics Center, Beijing \\ 100871, China
}

\begin{abstract}
This paper summarizes the change of astronomical observing conditions since 1986 at Huairou Solar Observing Station. Our results from 1988 to 1999 are as follows: quantitatively, stray light has increased and visibility has decreased; qualitatively, the seeing condition has become worse.
\end{abstract}

\section{Introduction}

Huairou Solar Observing Station (HSOS) is located on a small island near the north bank of Huairou Reservoir, $60 \mathrm{~km}$ north of Beijing. The water surrounding the station extends out about $2 \mathrm{~km}$ from the observing tower. The observing dome is on the top of a 31-meter tower. The elevation of HSOS is about $62 \mathrm{~m}$, and the location is $40 .^{\circ} 4 \mathrm{~N}$ and $116 .{ }^{\circ} 6 \mathrm{E}$. The mean temperature is $26^{\circ} \mathrm{C}$ in summer and $-4^{\circ} \mathrm{C}$ in winter. The annual precipitation is about $500 \mathrm{~mm}$.

The vector magnetic fields and line-of-sight velocity at the photosphere and chromosphere of the Sun are observed at HSOS. The main optical telescope is a Solar Multi-Channel Telescope (SMCT), which consists of a $60 \mathrm{~cm}$ Solar Nine-Channel Telescope, a $35 \mathrm{~cm}$ Solar Magnetic Field Telescope, a $10 \mathrm{~cm}$ Fulldisc Magnetograph, a $14 \mathrm{~cm}$ Partial and Full-disc $\mathrm{H} \alpha$ Telescope and a $8 \mathrm{~cm}$ Full-disc Calcium Monochromator. HSOS plays an important role in the solar community.

Solar observations at HSOS began in 1986 and routine observations began in 1988. In addition to the scientific data, we have also recorded some parameters of observational conditions, such as seeing, stray light and visibility of the terrestrial atmosphere, etc. In this paper, we give a summary of these parameters.

\section{Observations and Results}

\subsection{Amplification Factor}

In our solar observation, the image collecting and processing system consists of a CCD Camera and corresponding frame grabber. Before digitizing a solar image, 

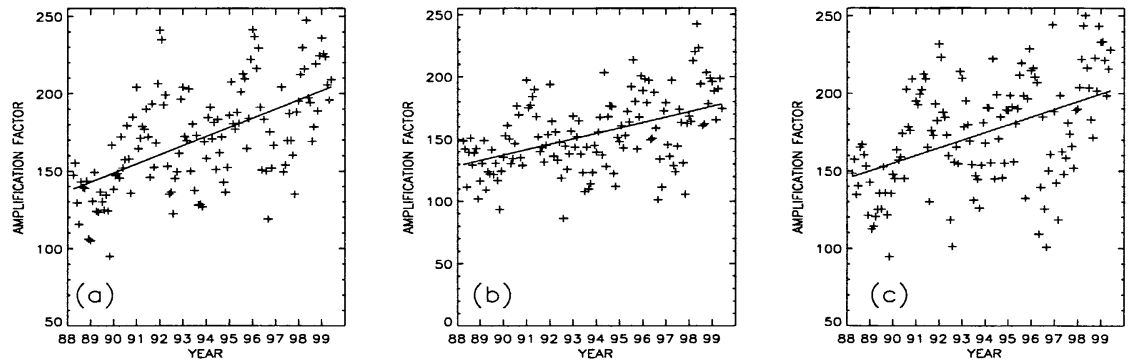

Figure 1. Change of amplification factor with time for HSOS solar observations. Each point is a monthly average over a daily time interval (a) times before $0200 \mathrm{UT}$, (b) $0200-0600 \mathrm{UT}$ and (c) after $0600 \mathrm{UT}$. (local noon $=0400 \mathrm{UT}$ )
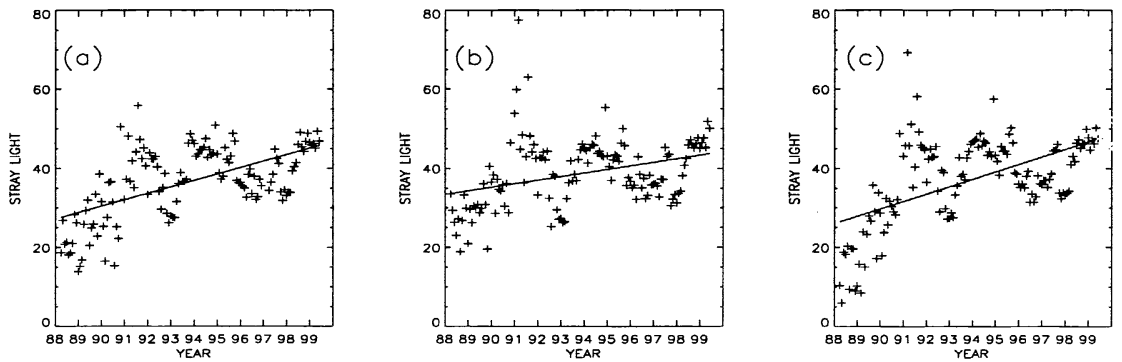

Figure 2. Change of stray light with time. Plots as in Fig. 1.

the amplification factor of the system must be determined by the intensity of received sunlight, in order to get highest accuracy. The weaker the intensity of sunlight, the larger the amplification factor and thus the larger the noise. Of course the annual change of solar declination causes a change of sunlight with the seasons, but it does not affect long-term statistics, such as we use in this paper. On the other hand, the terrestrial atmosphere can reduce sunlight by absorption, scattering, etc. Figure 1 shows the amplification factor from March 1988 to May 1999. Each point represents the average value within one month. It is obvious that the condition of the atmosphere has worsened in the past ten years. The solid lines are linear fits and have slopes indicating increases of $49 \%$, $36 \%$ and $41 \%$ for (a), (b) and (c) respectively.

\subsection{Stray Light}

Generally, the stray light in solar optical observation comes from the Sun itself, the terrestrial atmosphere and the telescope. For our long-term statistics, we might think that most of the change of stray light would be caused by the atmosphere. Figure 2 shows the stray light recorded in our system from March 1988 to May 1999. The increase of stray light is obvious. The slopes for the fits indicate increases of $14 \%, 8 \%$ and $17 \%$ for (a), (b) and (c) respectively. 


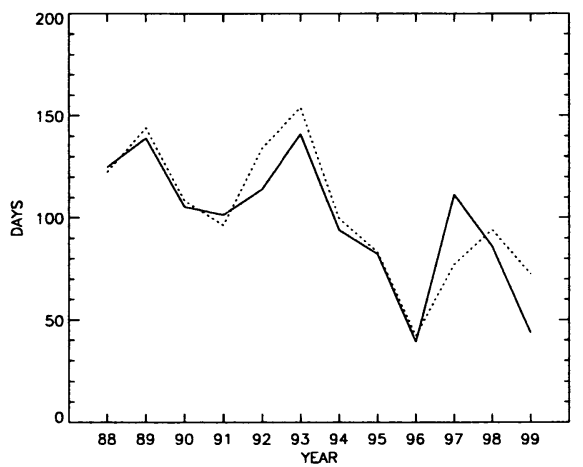

Figure 3. The annual number of days with "good" seeing conditions. Solid line: morning (before 0400 UT). Dotted line: afternoon (after 0400 UT).

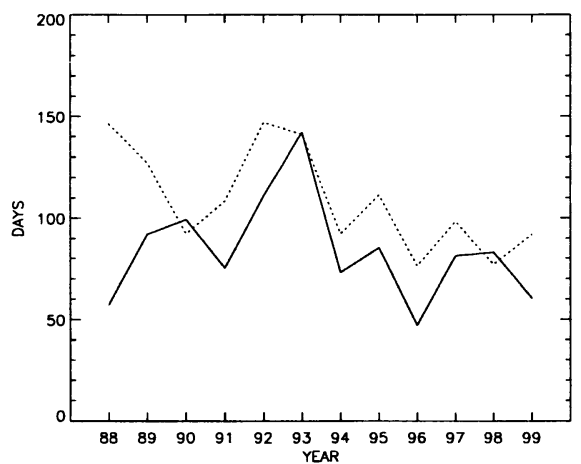

Figure 4. The annual number of days with "good" visibility. Lines as in Fig. 3.

\subsection{Seeing}

Every observational day we also record the seeing condition, as estimated by visual judgement. We divide seeing condition into six grades: best, better, good, bad, worse, worst. Figure 3 shows that the annual number of days with "good" grade or better seeing has declined significantly over the eleven years of records.

\subsection{Visibility}

The visibility of terrestrial atmosphere is also recorded by visual judgement. Compared with the amplification factor discussed earlier, this parameter is less strict but more direct. We also divide the visibility into six grades: best, better, good, bad, worse, worst. Figure 4 shows that the annual number of days with "good" grade or better visibility has tended to decline. 


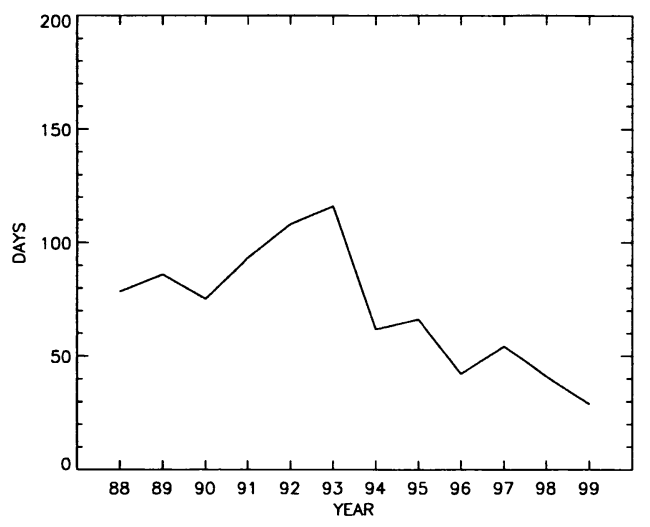

Figure 5. The annual number of days with "good" image quality.

\subsection{Image Quality}

Image quality is affected by all of the factors mentioned above. Visual judgement of this is also carried out every day. We again divide the image quality into the same six categories. Figure 5 shows the annual number of days with "good" grade or better image quality. One can see that the number of days with goodquality data has significantly declined.

\section{Discussion}

The observational conditions at HSOS are becoming worse. We think this serious change comes from either a global change of weather or from local air pollution. Here we give a simple discussion of local factors that affect the atmosphere. The first factor is the expansion of the city dimensions. By the official plan of Beijing, the construction volume will increase $100 \%$ from 1990 to 2000 and $200 \%$ to 2010. By the most conservative estimates, the expansion rate of Beijing in the past ten years has been even larger than this plan. The second factor is the increasing number of automobiles. Experts estimated that the number of automobiles in Beijing is now 1,400,000, several times more than ten years ago. They warn that this number is growing by 200,000 every year. In addition, there are some others factors that seriously pollute the local atmosphere, such as the exploitation of natural attractions for tours around Beijing, etc.

Are there ways to deal with this bad condition? The permanent way is to reduce pollution, but this is a long-term sociological problem. For our current observations, modern technology provides some opportunities, such as more sensitive detectors, adaptive optics, correlation trackers, etc. In fact, as has been done at HSOS, these tools have been widely applied in astronomy. This is successful, but at the cost of a lot of bills and technical complexity.

Acknowledgments. We thank Ms. Wang Guoping and Mr. Qi Hongwei for their assistance. 